\title{
PERIODIC ORBITS OF CONTINUOUS MAPPINGS OF THE CIRCLE
}

BY

LOUIS BLOCK

\begin{abstract}
Let $f$ be a continuous map of the circle into itself and let $P(f)$ denote the set of positive integers $n$ such that $f$ has a periodic point of period $n$. It is shown that if $1 \in P(f)$ and $n \in P(f)$ for some odd positive integer $n$ then for every integer $m>n, m \in P(f)$. Furthermore, if $P(f)$ is finite then there are integers $m$ and $n$ (with $m>1$ and $n>0$ ) such that $P(f)=\left\{m, 2 \cdot m, 4 \cdot m, 8 \cdot m, \ldots, 2^{n}\right.$. $m$ \}.
\end{abstract}

1. Introduction. Let $I$ denote a closed bounded interval on the real line and let $C^{0}(I, I)$ denote the space of continuous maps of $I$ into itself. For $f \in C^{0}(I, I)$, let $P(f)$ denote the set of all positive integers $n$ such that $f$ has a periodic point of period $n$ (see $\$ 2$ for definition). One may ask the following question. If $k \in P(f)$, what other integers must be elements of $P(f)$ ?

This question is answered by a theorem of Šarkovskii. Consider the following ordering of the positive integers:

$$
\begin{array}{r}
1,2,4,8, \ldots \ldots, 7 \cdot 8,5 \cdot 8,3 \cdot 8, \ldots, 7 \cdot 4,5 \cdot 4,3 \cdot 4, \ldots, \\
7 \cdot 2,5 \cdot 2,3 \cdot 2, \ldots, 7,5,3 .
\end{array}
$$

Sarkovskii's theorem states that if $n \in P(f)$ and $m$ is to the left of $n$ in the above ordering then $m \in P(f)$ (see [3] or [4]). Furthermore, if $m$ is to the right of $n$ in the above ordering, then there is a map $f \in C^{0}(I, I)$ with $n \in P(f)$ and $m \notin P(f)$.

In this paper we obtain some similar results in $C^{0}\left(S^{1}, S^{1}\right)$ (the space of continuous maps of the circle into itself). Since for any positive integer $n$, there is a map $f \in C^{0}\left(S^{1}, S^{1}\right)$ with $P(f)=\{n\}$ (where $P(f)$ is defined as above) one cannot obtain an ordering as in Sarkovskii's theorem. However, we do obtain the following result.

Theorem A. Let $f \in C^{0}\left(S^{1}, S^{1}\right)$. Suppose $1 \in P(f)$ and $n \in P(f)$ for some odd integer $n>1$. Then for every integer $m>n, m \in P(f)$.

We remark that if the hypothesis of Theorem $A$ is satisfied, it is possible that for every integer $k$ with $1<k<n, k \notin P(f)$ (see Proposition 12 in §5). Using Theorem A we obtain the following result which characterizes $P(f)$ when $P(f)$ is finite.

TheOrem B. Let $f \in C^{0}\left(S^{1}, S^{1}\right)$ and suppose that $P(f)$ is finite. Then there are integers $m$ and $n$ (with $m \geqslant 1$ and $n \geqslant 0$ ) such that $P(f)=\{m, 2 \cdot m, 4 \cdot m, 8$. $\left.m, \ldots, 2^{n} \cdot m\right\}$.

Received by the editors July 10, 1979.

1980 Mathematics Subject Classification. Primary 54H20.

(C) 1980 American Mathematical Society 0002-9947/80/0000-0364/\$03.50 
It is known that for any integers $m$ and $n$ with $m \geqslant 1$ and $n \geqslant 0$ there is a differentiable map $f$ of the circle with $P(f)=\left\{m, 2 \cdot m, 4 \cdot m, 8 \cdot m, \ldots, 2^{n} \cdot m\right\}$ (see [1]). Also, in [1], Theorem B is proved for a certain subset of $C^{0}\left(S^{1}, S^{1}\right)$.

Finally, note that Sarkovskii's theorem implies that for $f \in C^{0}(I, I)$ if $3 \in P(f)$ then $P(f)=N$, where $N$ denotes the set of positive integers. This result is also proved in [2]. It also follows from Sarkovskii's theorem that if $S \subset N$ with the property that for any $f \in C^{0}(I, I), S \subset P(f) \Rightarrow P(f)=N$, then $3 \in S$. In this paper we obtain the following analogous result for the circle. The first statement, of course, follows immediately from Theorem A.

Theorem C. Let $f \in C^{0}\left(S^{1}, S^{1}\right)$. If $\{1,2,3\} \subset P(f)$ then $P(f)=N$. Conversely, if $S \subset N$ with the property that for any $f \in C^{0}\left(S^{1}, S^{1}\right), S \subset P(f) \Rightarrow P(f)=N$, then $\{1,2,3\} \subset S$.

2. Preliminary definitions and results. Let $f \in C^{0}\left(S^{1}, S^{1}\right)$. For any $n \in N$, we define $f^{n}$ inductively by $f^{1}=f$ and $f^{n}=f \circ f^{n-1}$. Let $f^{0}$ denote the identity map of $S^{1}$.

Let $x \in S^{1} . x$ is said to be a fixed point of $f$ if $f(x)=x . x$ is said to be a periodic point of $f$ if $f^{n}(x)=x$ for some $n \in N$. In this case, the smallest element of $\left\{n \in N: f^{n}(x)=x\right\}$ is called the period of $x$.

We define the orbit of $x$ to be $\left\{f^{n}(x): n=0,1,2, \ldots\right\}$. If $x$ is a periodic point of period $n$, we say the orbit of $x$ is a periodic orbit of period $n$. In this case the orbit of $x$ contains exactly $n$ points, each of which is a periodic point of period $n$.

We will use the following notation throughout this paper.

Notation. Let $a \in S^{1}$ and $b \in S^{1}$ with $a \neq b$. We write $[a, b],(a, b),(a, b]$, or $[a, b)$ to denote the closed, open, or half-open interval from a counterclockwise to $b$.

We will also use the following definition.

Definition. Let $I$ and $J$ be proper closed intervals on $S^{1}$ and let $f \in C^{0}\left(S^{1}, S^{1}\right)$. We say I f-covers $J$ if for some closed interval $K \subset I, f(K)=J$.

We conclude this section by proving four lemmas, which use this definition.

Lemma 1. Let $I=[a, b]$ be a proper closed interval on $S^{1}$ and let $f \in C^{0}\left(S^{1}, S^{1}\right)$. Suppose $f(a)=c$ and $f(b)=d$ and $c \neq d$. Then either $I f$-covers $[c, d]$ or $I f$-covers $[d, c]$.

Proof. Let $A=\{x \in I: f(x)=c\}$. There is a point $v \in A$ such that $(v, b] \cap A$ $=\varnothing$. Let $B=\{x \in[v, b]: f(x)=d\}$. There is a point $w \in B$ such that $[v, w) \cap B$ $=\varnothing$.

We have $f(v)=c, f(w)=d$, and if $x \in(v, w)$ then $f(x) \notin\{c, d\}$. Hence, if $K=[v, w]$ then $f(K)=[c, d]$ or $f(K)=[d, c]$. Q.E.D.

Lemma 2. Let $f \in C^{0}\left(S^{1}, S^{1}\right)$. Let $I$ and $J$ be proper closed intervals on $S^{1}$ such that I f-covers $J$. Suppose $L$ is a closed interval with $L \subset J$. Then $I f$-covers $L$.

Proof. By hypothesis, there is a closed interval $K \subset I$ with $f(K)=J$. Let $L=[c, d]$. There are points $a \in K$ and $b \in K$ with $f(a)=c$ and $f(b)=d$. Let $K_{1}$ 
be the closed interval with endpoints $a$ and $b$ such that $K_{1} \subset K$. By Lemma 1, either $K_{1} f$-covers $[c, d]$ or $K_{1} f$-covers $[d, c]$. Since $K_{1} \subset K$ and $f(K)=J, K_{1}$ cannot $f$-cover $[d, c]$. Hence $K_{1} f$-covers $[c, d]$. Since $K_{1} \subset K \subset I, I f$-covers $[c, d]$. Q.E.D.

Lemma 3. Let $f \in C^{0}\left(S^{1}, S^{1}\right)$. Suppose $N$ is a proper closed interval on $S^{1}$ such that $N$-covers $N$. Then $f$ has a fixed point in $N$.

Proof. By hypothesis, for some closed interval $K \subset N, f(K)=N$. There are points $v \in K$ and $w \in K$ such that $f(v)$ and $f(w)$ are the two endpoints of $N$. Let $L$ be the closed interval with endpoints $v$ and $w$ such that $L \subset K$. By continuity, $f$ has a fixed point in $L$. Q.E.D.

LEMMA 4. Suppose $f \in C^{0}\left(S^{1}, S^{1}\right)$ and suppose $M_{1}, M_{2}, \ldots, M_{n}$ are proper closed intervals on $S^{1}$ such that $M_{k} f$-covers $M_{k+1}$ for $k=1, \ldots, n-1$, and $M_{n} f$-covers $M_{1}$. Then there is a fixed point $z$ of $f^{n}$ such that $z \in M_{1}, f(z) \in M_{2}, \ldots, f^{n-1}(z) \in$ $M_{n}$.

Proof. Since $M_{n} f$-covers $M_{1}$, there is an interval $J_{n} \subset M_{n}$ such that $f\left(J_{n}\right)=M_{1}$. Similarly, there are intervals $J_{1}, \ldots, J_{n-1}$ such that for each $k=1, \ldots, n-1$, $J_{k} \subset M_{k}$ and $f\left(J_{k}\right)=J_{k+1}$. It follows that $f^{n}\left(J_{1}\right)=M_{1}$. By the proof of Lemma 3, $f^{n}$ has a fixed point $z \in J_{1}$. Clearly $z \in M_{1}, f(z) \in M_{2}, \ldots, f^{n-1}(z) \in M_{n}$. Q.E.D.

\section{Proof of Theorem A.}

Definition. Let $f \in C^{0}\left(S^{1}, S^{1}\right)$ and let $P=\left\{p_{1}, \ldots, p_{n}\right\}$ be a periodic orbit of $f$ of period $n$. We say $P$ is labeled in order if for $k=1, \ldots, n-1, P \cap\left(p_{k}, p_{k+1}\right)=$ $\varnothing$, and $P \cap\left(p_{n}, p_{1}\right)=\varnothing$. In this case we define the intervals determined by $P$ to be the $n$ closed intervals $I_{1}=\left[p_{1}, p_{2}\right], I_{2}=\left[p_{2}, p_{3}\right], \ldots, I_{n-1}=\left[p_{n-1}, p_{n}\right], I_{n}=$ $\left[p_{n}, p_{1}\right]$.

Lemma 5. Let $f \in C^{0}\left(S^{1}, S^{1}\right)$. Let $P=\left\{p_{1}, \ldots, p_{n}\right\}$ be a periodic orbit of $f$ of odd period $n \geqslant 3$. Suppose $P$ is labeled in order and let $I_{1}, \ldots, I_{n}$ be the intervals determined by $P$. Suppose that for some $j$ and $k$ with $j \in\{1, \ldots, n\}$ and $k \in$ $\{1, \ldots, n\}, I_{i}$ does not $f$-cover $I_{j}$ for all $i \in\{1, \ldots, n\}$ with $i \neq j$, and $I_{i}$ does not f-cover $I_{k}$ for all $i \in\{1, \ldots, n\}$ with $i \neq k$. Then $j=k$.

Proof. Suppose $j \neq k$. Let $v$ be a point in the interior of $I_{k}$, and let $w$ be a point in the interior of $I_{j}$. Then $v \neq w$. Let $A=P \cap(v, w)$ and $B=P \cap(w, v)$. Then $A \neq \varnothing, B \neq \varnothing, A \cap B=\varnothing$ and $A \cup B=P$.

If $f(x) \in A$ for some $x \in A$ then by hypothesis and Lemma $1, f(A) \subset A$. This is impossible, since $P$ is a periodic orbit. Thus, $f(x) \notin A$ for all $x \in A$. Hence, $f(A) \subset B$. Similarly it follows that $f(B) \subset A$.

Since $f$ maps $P$ onto $P$ it follows that $f(A)=B$ and $f(B)=A$. Thus, $A$ and $B$ have the same number of elements. Since $A \cup B=P$ and $A \cap B=\varnothing$, this contradicts the fact that ' has an odd number of elements. Q.E.D. 
Lemma 6. Suppose $f \in C^{0}\left(S^{1}, S^{1}\right)$ and $f$ has a periodic orbit $P=\left\{p_{1}, \ldots, p_{n}\right\}$ of odd period $n \geqslant 3$. Suppose $P$ is labeled in order, and let $I_{1}, \ldots, I_{n}$ be the intervals determined by $P$. Suppose also that $f$ has a fixed point $e$. Then $f$ has a fixed point $z$ with the property that if $I_{k}$ is the interval determined by $P$ with $z \in I_{k}$, there is some $j \in\{1, \ldots, n\}$ with $j \neq k$ such that $I_{j} f$-covers $I_{k}$.

Proof. Without loss of generality we may assume that $e \in I_{n}$. We may also assume that for each $j \in\{1, \ldots, n-1\}, I_{j}$ does not $f$-cover $I_{n}$ (or else the conclusion of the lemma holds with $z=e$ ).

Let $m$ be the smallest positive integer such that if $f\left(p_{m}\right)=p_{r}$ then $r<m$. Note that $2 \leqslant m \leqslant n$, so $1 \leqslant m-1 \leqslant n-1$. In particular $m-1 \neq n$.

Since $I_{m-1}$ does not $f$-cover $I_{n}$, it follows from Lemmas 1 and 2 that $I_{m-1}$ $f$-covers $I_{m-1}$. By Lemma 3, $f$ has a fixed point $z \in I_{m-1}$. Since $I_{j}$ does not $f$-cover $I_{n}$ for all $j \in\{1, \ldots, n-1\}$, it follows from Lemma 5 that for some $j \in$ $\{1, \ldots, n\}$ with $j \neq m-1, I_{j} f$-covers $I_{m-1}$. Q.E.D.

Lemma 7. Let $f \in C^{0}\left(S^{1}, S^{1}\right)$ and let $P$ be a periodic orbit of $f$ of period $m$ where $m \geqslant 3$. Suppose that $\left\{M_{1}, \ldots, M_{k}\right\}$ is a collection of closed intervals with $2 \leqslant k \leqslant$ m such that

(1) for each $j \in\{1, \ldots, k\}$, there are no elements of $P$ in the interior of $M_{j}$,

(2) if $i \neq j, M_{i}$ and $M_{j}$ have disjoint interiors,

(3) if $j \in\{2, \ldots, k\}$ the endpoints of $M_{j}$ are in $P$,

(4) if $b$ is an endpoint of $M_{1}$, either $b \in P$ or $b$ is a fixed point of $f$,

(5) for each $j \in\{1, \ldots, k-1\}, M_{j} f$-covers $M_{j+1}$,

(6) $M_{1} f$-covers $M_{1}$ and $M_{k} f$-covers $M_{1}$.

Then for any positive integer $n>k, f$ has a periodic point of period $n$.

Proof. Let $n>k$. We may assume $n \neq m$ since, by hypothesis, $f$ has a periodic point of period $m$.

Let $L_{1}=M_{1}, L_{2}=M_{1}, \ldots, L_{n-k}=M_{1}, L_{n-k+1}=M_{1}, L_{n-k+2}=M_{2}, L_{n-k+3}$ $=M_{3}, \ldots, L_{n-k+k}=L_{n}=M_{k}$. By Lemma 4 (applied to $\left.L_{1}, L_{2}, \ldots, L_{n}\right)$, there is a fixed point $z$ of $f^{n}$ such that $z \in L_{1}, f(z) \in L_{2}, \ldots, f^{n-1}(z) \in L_{n}$. Since $z \in M_{1}$ and $f^{n-k+1}(z) \in M_{2}$, it follows from (2) and (3) of the hypothesis that $z$ is not a fixed point of $f$.

We claim that $z \notin P$. To prove this, first suppose that $n \geqslant k+2$. Then $L_{1}=L_{2}$ $=L_{3}=M_{1}$. Hence $z \in M_{1}, f(z) \in M_{1}$ and $f^{2}(z) \in M_{1}$. Since $P$ is a periodic orbit of period $m \geqslant 3$, it follows from (1) of the hypothesis that $z \notin P$. Now, suppose that $n<k+2$. Then $n<m+2$. Since $n \neq m$ and $m \geqslant 3, n$ is not a multiple of $m$. Since $f^{n}(z)=z$, it follows that $z \notin P$.

Since $z$ is not a fixed point of $f$ and $z \notin P$ it follows from (4) of the hypothesis that $z$ is in the interior of $M_{1}$. Also, since $f^{n}(z)=z \notin P$, for any positive integer $r<n, f^{r}(z) \notin P$ (and $f^{r}(z)$ is not a fixed point of $f$ ). Thus, by (3) and (4), for any positive integer $r<n, f^{r}(z)$ is not an endpoint of any of the intervals $M_{1}, \ldots, M_{k}$. It follows from this, and (2), and the fact that $z \in M_{1}, f(z) \in M_{1}, f^{2}(z) \in$ $M_{1}, \ldots, f^{n-k}(z) \in M_{1}, f^{n-k+1}(z) \in M_{2}, \ldots, f^{n-1}(z) \in M_{k}$, that $z$ is a periodic point of $f$ of period $n$. Q.E.D. 
Theorem A. Suppose $f \in C^{0}\left(S^{1}, S^{1}\right)$. Suppose $1 \in P(f)$ and $n \in P(f)$ for some odd integer $n>1$. Then for every integer $m>n, m \in P(f)$.

Proof. By hypothesis $f$ has a periodic orbit $P=\left\{p_{1}, \ldots, p_{n}\right\}$ of period $n$. Without loss of generality we may assume that $P$ is labeled in order. Let $I_{1}, \ldots, I_{n}$ be the intervals determined by $P$.

Also, by hypothesis, $f$ has a fixed point $e$. We may assume without loss of generality that $e \in I_{n}$. By Lemma 6 , we may also assume that for some $j \in$ $\{1, \ldots, n-1\}, I_{j} f$-covers $I_{n}$.

Let $f\left(p_{1}\right)=p_{s}$ and $f\left(p_{n}\right)=p_{t}$. We have two cases.

Case 1. Either $\left[e, p_{1}\right] f$-covers $\left[e, p_{s}\right]$ or $\left[p_{n}, e\right] f$-covers $\left[p_{t}, e\right]$.

Since these are analogous we may assume $\left[e, p_{1}\right] f$-covers $\left[e, p_{s}\right]$. Thus, by Lemma $2,\left[e, p_{1}\right] f$-covers $\left[e, p_{1}\right]$, and $\left[e, p_{1}\right] f$-covers each interval $I_{j}$ with $j \in\{1, \ldots, s-$ $1\}$.

Suppose that for some $j \in\{1, \ldots, s-1\}, I_{j} f$-covers $I_{n}$. Then the hypothesis of Lemma 7 is satisfied with $k=2, M_{1}=\left[e, p_{1}\right]$, and $M_{2}=I_{j}$. Hence, by Lemma 7 , the conclusion of the theorem holds.

Thus, we may assume that for all $j \in\{1, \ldots, s-1\}, I_{j}$ does not $f$-cover $I_{n}$. Since $I_{j} f$-covers $I_{n}$ for some $j \in\{1, \ldots, n-1\}$, this implies that $s-1<n-1$. Hence, $s<n$.

Since $s<n$, for some integer $r$ with $2 \leqslant r \leqslant s, f\left(p_{r}\right) \notin\left\{p_{1}, \ldots, p_{s}\right\}$. We may assume, by choosing $r$ smaller if necessary that $f\left(p_{r-1}\right) \in\left\{p_{1}, \ldots, p_{s}\right\}$. Let $f\left(p_{r}\right)=$ $p_{q}$. Since $I_{r-1}$ does not $f$-cover $I_{n}$, by Lemmas 1 and $2, I_{r-1} f$-covers $\left[f\left(p_{r-1}\right), p_{q}\right]$. Hence, for every positive integer $j$ with $s \leqslant j \leqslant q-1, I_{r-1} f$-covers $I_{j}$.

Note that by choice of $p_{r}$ and $p_{q}, s \leqslant q-1$. Suppose that for some positive integer $j$ with $s \leqslant j \leqslant q-1, I_{j} f$-covers $I_{n}$. Then the conclusion of the theorem holds by Lemma 7 (with $k=3, M_{1}=\left[e, p_{1}\right], M_{2}=I_{r-1}$ and $M_{3}=I_{j}$ ).

By repeating the argument of the preceding three paragraphs at most $n$ times, using the fact that for some $j \in\{1, \ldots, n-1\}, I_{j} f$-covers $I_{n}$, eventually we obtain a collection of closed intervals $\left\{M_{1}, M_{2}, \ldots, M_{k}\right\}$ with $k \leqslant n$, such that the hypothesis of Lemma 7 is satisfied. Thus, the conclusion of the theorem follows from Lemma 7.

Case 2. $\left[e, p_{1}\right]$ does not $f$-cover $\left[e, p_{s}\right]$ and $\left[p_{n}, e\right]$ does not $f$-cover $\left[p_{t}, e\right]$.

By Lemma $1,\left[e, p_{1}\right] f$-covers $\left[p_{s}, e\right]$ and $\left[p_{n}, e\right] f$-covers $\left[e, p_{t}\right]$. We claim that $I_{n}=\left[p_{n}, p_{1}\right] f$-covers $I_{n}$. To prove this, note that since $\left[e, p_{1}\right] f$-covers $\left[p_{s}, e\right]$, there is a point $a \in\left(e, p_{1}\right]$ such that $f(a)=p_{n}$ but $f(x) \neq p_{n}$ for all $x \in(e, a)$. Since $f(e)=e$ and $f(a)=p_{n},[e, a] f$-covers $\left[e, p_{n}\right]$ or $[e, a] f$-covers $\left[p_{n}, e\right]$. Since $\left[e, p_{1}\right]$ does not $f$-cover $\left[e, p_{s}\right],[e, a]$ does not $f$-cover $\left[e, p_{s}\right]$. By Lemma $2,[e, a]$ does not $f$-cover $\left[e, p_{n}\right]$. Hence, $[e, a] f$-covers $\left[p_{n}, e\right]$. In particular $f([e, a]) \supset\left[p_{n}, e\right]$.

Suppose that for some $z \in(e, a), f(z) \notin\left[p_{n}, p_{1}\right]$. Since $f(z) \notin\left[p_{n}, p_{1}\right]$ and $f(e) \in$ $\left[p_{n}, p_{1}\right]$, by continuity, for some $q \in(e, a), f(q)=p_{1}$ or $f(q)=p_{n}$. Since $q \in(e, a)$ it follows from the choice of $a$ that $f(q) \neq p_{n}$. Hence $f(q)=p_{1}$. Now, it follows from the choice of $a$, that $f([e, a])$ is a proper closed interval on $S^{1}$ and $p_{n}$ is an endpoint of $f([e, a])$. Also, $e \in f([e, a])$ and $p_{1} \in f([e, a])$. Hence, either $\left[p_{n}, p_{1}\right] \subset$ $f([e, a])$ or $\left[e, p_{n}\right] \subset f([e, a])$. If $\left[e, p_{n}\right] \subset f([e, a])$, it follows as in the proof of 
Lemma 2 , using the fact that $f([e, a]) \neq S^{1}$, that $[e, a] f$-covers $\left[e, p_{n}\right]$. This implies, by Lemma 2 , that $[e, a] f$-covers $\left[e, p_{s}\right]$. Thus, $\left[e, p_{1}\right] f$-covers $\left[e, p_{s}\right]$, a contradiction. Hence $\left[p_{n}, p_{1}\right] \subset f([e, a])$. Since $f([e, a]) \neq S^{1}$, this implies that $[e, a] f$-covers $\left[p_{n}, p_{1}\right]$. Thus, $\left[p_{n}, p_{1}\right] f$-covers $\left[p_{n}, p_{1}\right]$.

We have shown that our claim holds if $f(z) \notin\left[p_{n}, p_{1}\right]$ for some $z \in(e, a)$. Hence, we may assume that $f([e, a]) \subset\left[p_{n}, p_{1}\right]$.

Since $\left[p_{n}, e\right] f$-covers $\left[e, p_{t}\right]$, there is a point $b \in\left[p_{n}, e\right)$ such that $f(b)=p_{1}$ but $f(x) \neq p_{1}$ for all $x \in(b, e)$. It follows that $f([b, e]) \supset\left[e, p_{1}\right]$ (by the same argument used to show $\left.f([e, a]) \supset\left[p_{n}, e\right]\right)$. Also, we may assume that $f([b, e]) \subset\left[p_{n}, p_{1}\right]$ (by the same argument used to show that we may assume that $\left.f([e, a]) \subset\left[p_{n}, p_{1}\right]\right)$. Thus, $f([b, a])=\left[p_{n}, p_{1}\right]$. Since $[b, a] \subset\left[p_{n}, p_{1}\right]$, this establishes our claim that $\left[p_{n}, p_{1}\right] f$-covers $\left[p_{n}, p_{1}\right]$.

Now, since $\left[e, p_{1}\right] f$-covers $\left[p_{s}, e\right],\left[p_{n}, p_{1}\right] f$-covers $\left[p_{s}, e\right]$. Also, since $\left[p_{n}, e\right]$ $f$-covers $\left[e, p_{t}\right],\left[p_{n}, p_{1}\right] f$-covers $\left[e, p_{t}\right]$. Hence, by Lemma 2 , for any integer $j$ with $1 \leqslant j \leqslant t-1$ or $s \leqslant j \leqslant n-1,\left[p_{n}, p_{1}\right] f$-covers $I_{j}$.

Suppose that for some integer $j$ with $1 \leqslant j \leqslant t-1$ or $s \leqslant j \leqslant n-1, I_{j} f$-covers $I_{n}$. Then the conclusion of the theorem holds by Lemma 7 (with $k=2, M_{1}=I_{n}$ and $M_{2}=I_{j}$ ). Hence, we may assume that for every integer $j$ with $1 \leqslant j \leqslant t-1$ or $s \leqslant j \leqslant n-1, I_{j}$ does not $f$-cover $I_{n}$. Since $I_{j} f$-covers $I_{n}$ for some integer $j$ with $1 \leqslant j \leqslant n-1$, this implies that $t<s$.

Note that we cannot have both $f\left(\left\{p_{1}, \ldots, p_{t}\right\}\right) \subset\left\{p_{s}, \ldots, p_{n}\right\}$ and $f\left(\left\{p_{s}, \ldots, p_{n}\right\}\right) \subset\left\{p_{1}, \ldots, p_{t}\right\}$. This follows from the fact that $\left\{p_{1}, \ldots, p_{n}\right\}$ is a periodic orbit and $t<s$, and uses the fact that $n$ is odd in the case $t=s-1$. Without loss of generality we may assume that $f\left(\left\{p_{1}, \ldots, p_{t}\right\}\right)$ is not a subset of $\left\{p_{s}, \ldots, p_{n}\right\}$.

Let $w$ be the smallest positive integer such that $f\left(p_{w}\right) \notin\left\{p_{s}, \ldots, p_{n}\right\}$. Then $2 \leqslant w \leqslant t$ and $I_{w-1} f$-covers the interval $\left[f\left(p_{w}\right), f\left(p_{w-1}\right)\right]$ (since $I_{w-1}$ does not $f$-cover $\left.I_{n}\right)$. Hence $I_{w-1} f$-covers the interval $\left[f\left(p_{w}\right), p_{s}\right]$. Let $f\left(p_{w}\right)=p_{v}$. Then $v \leqslant s-1$ and $I_{w-1} f$-covers each interval $I_{j}$ with $v \leqslant j \leqslant s-1$.

Suppose for some integer $j$ with $v \leqslant j \leqslant s-1, I_{j} f$-covers $I_{n}$. Then the conclusion of the theorem holds by Lemma 7 (with $k=3, M_{1}=I_{n}, M_{2}=I_{w-1}$ and $M_{3}=I_{j}$ ).

By repeating the argument of the preceding four paragraphs at most $n$ times, using the fact that for some $j \in\{1, \ldots, n-1\} I_{j} f$-covers $I_{n}$, we eventually obtain a collection of closed intervals $\left\{M_{1}, M_{2}, \ldots, M_{k}\right\}$ with $k \leqslant n$ such that the hypothesis of Lemma 7 is satisfied. Thus, the conclusion of the theorem follows from Lemma 7. Q.E.D.

\section{Proof of Theorem B.}

Corollary 8. Let $f \in C^{0}\left(S^{1}, S^{1}\right)$. Suppose $m \in P(f)$ and $n \in P(f)$ where $m$ and $n$ are odd integers with $m \neq n$. Then $P(f)$ is infinite.

Proof. Without loss of generality, we may assume that $m<n$. Then $1 \in P\left(f^{m}\right)$ and for some odd integer $k>1, k \in P\left(f^{m}\right)$. By Theorem $\mathrm{A}, P\left(f^{m}\right)$ is infinite. Hence, $P(f)$ is infinite. Q.E.D. 
Corollary 9. Suppose $f \in C^{0}\left(S^{1}, S^{1}\right)$ and $P(f)$ is finite. Then for some integers $m$ and $n$ with $m \geqslant 1$ and $n \geqslant 0, P(f) \subset\left\{m, 2 \cdot m, 4 \cdot m, 8 \cdot m, \ldots, 2^{n} \cdot m\right\}$.

Proof. Let $m$ be the smallest element of $P(f)$. Since $P(f)$ is finite, it suffices to prove that if $k \in P(f)$ then $k=2^{i} \cdot m$ for some nonnegative integer $i$.

Let $k \in P(f)$. Let $m=2^{r} \cdot s$ where $s$ is odd, $s \geqslant 1$ and $r \geqslant 0$, and let $k=2^{v} \cdot w$ where $w$ is odd, $w \geqslant 1$ and $v \geqslant 0$. Let $j$ be the largest element of $\left\{2^{r}, 2^{v}\right\}$. Then $P\left(f^{j}\right)$ is finite, $s \in P\left(f^{j}\right), w \in P\left(f^{j}\right)$ and $s$ and $w$ are odd. By Corollary $8, s=w$.

Since $m$ is the smallest element of $P(f)$ and $s=w$, we have $v \geqslant r$. Let $i=v-r$. Then $i \geqslant 0$ and $k=2^{v} \cdot w=2^{v} \cdot s=2^{v-r} \cdot 2^{r} \cdot s=2^{i} \cdot m$. Q.E.D.

Lemma 10. Let $f \in C^{0}\left(S^{1}, S^{1}\right)$ and suppose that $\left\{p_{1}, p_{2}, p_{3}, p_{4}\right\}$ is a periodic orbit of $f$ of period 4 , labeled in order. Suppose that one of the following holds.

(i) $f\left(p_{1}\right)=p_{2}, f\left(p_{2}\right)=p_{3}, f\left(p_{3}\right)=p_{4}, f\left(p_{4}\right)=p_{1}$.

(ii) $f\left(p_{1}\right)=p_{4}, f\left(p_{4}\right)=p_{3}, f\left(p_{3}\right)=p_{2}, f\left(p_{2}\right)=p_{1}$.

Suppose also that $1 \in P(f)$. Then $5 \in P(f)$.

Proof. Since (i) and (ii) are analogous, we may assume that (i) holds. Let $I_{1}, I_{2}$, $I_{3}$ and $I_{4}$ be the intervals determined by $\left\{p_{1}, p_{2}, p_{3}, p_{4}\right\}$.

By Lemma 1, either $I_{1} f$-covers $I_{2}$ or $I_{1} f$-covers $I_{1} \cup I_{3} \cup I_{4}$. Suppose $I_{1} f$-covers $I_{1} \cup I_{3} \cup I_{4}$. If $I_{4} f$-covers $I_{1}$, then it follows from Lemma 7 (with $k=2, M_{1}=I_{1}$ and $\left.M_{2}=I_{4}\right)$ that $5 \in P(f)$. Also, if $I_{3} f$-covers $I_{1}$, then it follows from Lemma 7 (with $k=2, M_{1}=I_{1}$ and $M_{2}=I_{3}$ ) that $5 \in P(f)$. Hence we may assume that $I_{4}$ does not $f$-cover $I_{1}$ and $I_{3}$ does not $f$-cover $I_{1}$. This implies (by Lemma 1) that $I_{4}$ $f$-covers $I_{2} \cup I_{3} \cup I_{4}$ and $I_{3} f$-covers $I_{4}$. Hence, by Lemma 7 (with $k=2, M_{1}=I_{4}$ and $\left.M_{2}=I_{3}\right), 5 \in P(f)$.

Thus we may assume that $I_{1} f$-covers $I_{2}$. Similarly, we may assume that $I_{2}$ $f$-covers $I_{3}, I_{3} f$-covers $I_{4}$ and $I_{4} f$-covers $I_{1}$.

By hypothesis $f$ has a fixed point $e$. Without loss of generality we may assume that $e \in I_{4}$. Let $I_{4 \mathrm{~A}}=\left[p_{4}, e\right]$ and let $I_{4 \mathrm{~B}}=\left[e, p_{1}\right]$.

By Lemma 1 , either $I_{4 \mathrm{~B}} f$-covers $I_{4 \mathrm{~B}} \cup I_{1}$ or $I_{4 \mathrm{~B}} f$-covers $I_{2} \cup I_{3} \cup I_{4 \mathrm{~A}}$. If $I_{4 \mathrm{~B}}$ $f$-covers $I_{4 \mathrm{~B}} \cup I_{1}$, then it follows from Lemma 7 (with $k=4, M_{1}=I_{4 \mathrm{~B}}, M_{2}=I_{1}$, $M_{3}=I_{2}$ and $M_{4}=I_{3}$ ) that $5 \in P(f)$. Hence, we may assume that $I_{4 \mathrm{~B}} f$-covers $I_{2} \cup I_{3} \cup I_{4 \mathrm{~A}}$.

Also, by Lemma 1 , either $I_{4 \mathrm{~A}} f$-covers $I_{4 \mathrm{~B}}$ or $I_{4 \mathrm{~A}} f$-covers $I_{1} \cup I_{2} \cup I_{3} \cup I_{4 \mathrm{~A}}$. If $I_{4 \mathrm{~A}} f$-covers $I_{1} \cup I_{2} \cup I_{3} \cup I_{4 \mathrm{~A}}$, then it follows from Lemma 7 (with $k=2$, $M_{1}=I_{4 \mathrm{~A}}$ and $M_{2}=I_{3}$ ) that $5 \in P(f)$. Hence, we may assume that $I_{4 \mathrm{~A}} f$-covers $I_{4 \mathrm{~B}}$.

Now, we have that $I_{4 \mathrm{~A}} f$-covers $I_{4 \mathrm{~B}}, I_{4 \mathrm{~B}} f$-covers $I_{3}$ and $I_{3} f$-covers $I_{4 \mathrm{~B}}$. By Lemma 4 , there is a fixed point $z$ of $f^{3}$ such that $z \in I_{4 \mathrm{~A}}, f(z) \in I_{4 \mathrm{~B}}$ and $f^{2}(z) \in I_{3}$. Since $I_{4 \mathrm{~A}} \cap I_{4 \mathrm{~B}} \cap I_{3}=\varnothing, z$ is not a fixed point of $f$. Hence, $3 \in P(f)$. Thus, by Theorem A, $5 \in P(f)$. Q.E.D.

Lemma 11. Let $f \in C^{0}\left(S^{1}, S^{1}\right)$. Suppose $1 \in P(f), 4 \in P(f)$ and $5 \notin P(f)$. Then $2 \in P(f)$. 
Proof. Let $\left\{p_{1}, p_{2}, p_{3}, p_{4}\right\}$ be a periodic orbit of $f$ of period 4 , labeled in order. By Lemma 10, one of the following must hold.

(i) $f\left(p_{1}\right)=p_{3}, f\left(p_{3}\right)=p_{2}, f\left(p_{2}\right)=p_{4}$ and $f\left(p_{4}\right)=p_{1}$.

(ii) $f\left(p_{1}\right)=p_{3}, f\left(p_{3}\right)=p_{4}, f\left(p_{4}\right)=p_{2}$ and $f\left(p_{2}\right)=p_{1}$.

(iii) $f\left(p_{1}\right)=p_{4}, f\left(p_{4}\right)=p_{2}, f\left(p_{2}\right)=p_{3}$ and $f\left(p_{3}\right)=p_{1}$.

(iv) $f\left(p_{1}\right)=p_{2}, f\left(p_{2}\right)=p_{4}, f\left(p_{4}\right)=p_{3}$ and $f\left(p_{3}\right)=p_{1}$.

Note that (ii) is analogous to (i), because if (ii) holds and we let $q_{1}=p_{4}, q_{2}=p_{1}$, $q_{3}=p_{2}$ and $q_{4}=p_{3}$ then $f\left(q_{1}\right)=q_{3}, f\left(q_{3}\right)=q_{2}, f\left(q_{2}\right)=q_{4}$ and $f\left(q_{4}\right)=q_{1}$. Also, (iii) is analogous to (i) because if (iii) holds and we let $q_{1}=p_{4}, q_{2}=p_{3}, q_{3}=p_{2}$ and $q_{4}=p_{1}$ then $f\left(q_{1}\right)=q_{3}, f\left(q_{3}\right)=q_{2}, f\left(q_{2}\right)=q_{4}$ and $f\left(q_{4}\right)=q_{1}$. Finally, (iv) is analogous to (i), because if (iv) holds and we let $q_{1}=p_{2}, q_{2}=p_{3}, q_{3}=p_{4}$ and $q_{4}=p_{1}$ then $f\left(q_{1}\right)=q_{3}, f\left(q_{3}\right)=q_{2}, f\left(q_{2}\right)=q_{4}$ and $f\left(q_{4}\right)=q_{1}$. Hence, we may assume that (i) holds.

We claim that $I_{1} f$-covers $I_{3}$. Suppose that $I_{1}$ does not $f$-cover $I_{3}$. By Lemma $1, I_{1}$ $f$-covers $I_{4} \cup I_{1} \cup I_{2}$. If $I_{2} f$-covers $I_{4} \cup I_{1}$ then we obtain a contradiction (to the faci that $5 \notin P(f)$ ) by Lemma 7 (with $k=2, M_{1}=I_{1}$ and $M_{2}=I_{2}$ ). Hence, by Lemma $1, I_{2} f$-covers $I_{2} \cup I_{3}$. Also, if $I_{3} f$-covers $I_{1}$ then we obtain a contradiction by Lemma 7 (with $k=3, M_{1}=I_{1}, M_{2}=I_{2}, M_{3}=I_{3}$ ). Hence, by Lemma $1, I_{3}$ $f$-covers $I_{2} \cup I_{3} \cup I_{4}$. Again, by Lemma 7 (with $k=2, M_{1}=I_{2}, M_{2}=I_{3}$ ) we obtain a contradiction. This establishes our claim that $I_{1} f$-covers $I_{3}$.

We claim also that $I_{3} f$-covers $I_{1}$. Suppose that $I_{3}$ does not $f$-cover $I_{1}$. Then $I_{3}$ $f$-covers $I_{2} \cup I_{3} \cup I_{4}$ by Lemma 1 . If $I_{2} f$-covers $I_{2} \cup I_{3}$ then we obtain a contradiction by Lemma 7. Hence, by Lemma $1, I_{2} f$-covers $I_{4} \cup I_{1}$. Since $I_{1}$ $f$-covers $I_{3}$, we again obtain a contradiction by Lemma 7 (with $k=3, M_{1}=I_{3}$, $M_{2}=I_{2}$ and $\left.M_{3}=I_{1}\right)$. This establishes our claim that $I_{3} f$-covers $I_{1}$.

We have shown that $I_{1} f$-covers $I_{3}$ and $I_{3} f$-covers $I_{1}$. Since $I_{1} \cap I_{3}=\varnothing$, it follows from Lemma 4 that $2 \in P(f)$. Q.E.D.

Theorem B. Let $f \in C^{0}\left(S^{1}, S^{1}\right)$ and suppose that $P(f)$ is finite. Then there are integers $m$ and $n$ (with $m \geqslant 1$ and $n \geqslant 0$ ) such that $P(f)=\{m, 2 \cdot m, 4 \cdot m, 8$. $\left.m, \ldots, 2^{n} \cdot m\right\}$.

Proof. Let $m$ be the smallest element of $P(f)$ and let $k$ be the largest element of $P(f)$. By Corollary $9, k=2^{n} \cdot m$ for some nonnegative integer $n$ and $P(f) \subset\{m, 2$ $\left.\cdot m, 4 \cdot m, 8 \cdot m, \ldots, 2^{n} \cdot m\right\}$.

If $n=0$ or $n=1$ the conclusion of the theorem follows immediately, so we may assume that $n \geqslant 2$. Let $r=2^{n-2}$. Then $1 \in P\left(f^{r \cdot m}\right)$ and $4 \in P\left(f^{r \cdot m}\right)$. Also, since $P(f)$ is finite, $P\left(f^{r \cdot m}\right)$ is finite. Hence, by Theorem A, $5 \notin P\left(f^{r \cdot m}\right)$. By Lemma 11 , $2 \in P\left(f^{r \cdot m}\right)$. It follows from this, and the fact that $P(f) \subset\{m, 2 \cdot m, 4 \cdot m, 8$. $\left.m, \ldots, 2^{n} \cdot m\right\}$, that $2^{n-1} \cdot m \in P(f)$.

Now, if $n=2$ the conclusion of the theorem follows immediately. If $n>2$, it follows by the argument of the preceding paragraph (with $r=2^{n-3}$ instead of $r=2^{n-2}$ ) that $2^{n-2} \cdot m \in P(f)$. Thus, it follows by using this argument inductively, that $P(f)=\left\{m, 2 \cdot m, 4 \cdot m, 8 \cdot m, \ldots, 2^{n} \cdot m\right\}$. Q.E.D. 


\section{Proof of Theorem C.}

Proposition 12. Let $n$ be an integer with $n \geqslant 3$. There is a map $f \in C^{0}\left(S^{1}, S^{1}\right)$ such that $1 \in P(f)$ and $n \in P(f)$, but for every integer $k$ with $1<k<n, k \notin P(f)$.

Proof. Let $f \in C^{0}\left(S^{1}, S^{1}\right)$ with the following properties.

(1) $f$ has a periodic orbit $\left\{p_{1}, \ldots, p_{n}\right\}$ of period $n$, labeled in order, with $f\left(p_{i}\right)=p_{i+1}$ for $i=1, \ldots, n-1$, and $f\left(p_{n}\right)=p_{1}$.

(2) $f\left(I_{j}\right)=I_{j+1}$ for $j \in\{1, \ldots, n-1\}$, where $I_{1}, \ldots, I_{n}$ are the intervals determined by $\left\{p_{1}, \ldots, p_{n}\right\}$.

(3) $f$ has a fixed point $e \in I_{n}$.

(4) $f\left(\left[p_{n}, e\right]\right)=\left[e, p_{1}\right]$ and $f\left(\left[e, p_{1}\right]\right)=\left[e, p_{2}\right]$.

(5) For any $x \in\left(e, p_{1}\right), f(x) \neq x$ and $(x, f(x)) \subset\left(e, p_{1}\right)$.

By construction $1 \in P(f)$ and $n \in P(f)$. Also, by construction, if $x \in S^{1}$ such that $e$ is not in the orbit of $x$, then for any $j \in\{1, \ldots, n\}$, there is a point in the orbit of $x$ in $I_{j}$. Thus, for every integer $k$ with $1<k<n, k \notin P(f)$. Q.E.D.

LEMMA 13. There is a map $f \in C^{0}\left(S^{1}, S^{1}\right)$ such that for every integer $n>1$, $n \in P(f)$, but $1 \notin P(f)$.

Proof. Let $f \in C^{0}\left(S^{1}, S^{1}\right)$ with the following properties:

(1) $f$ has a periodic orbit $\left\{p_{1}, p_{2}, p_{3}\right\}$, labeled in order, with $f\left(p_{1}\right)=p_{2}, f\left(p_{2}\right)=p_{3}$ and $f\left(p_{3}\right)=p_{1}$.

(2) There are points $c_{1} \in\left(p_{1}, p_{2}\right), c_{2} \in\left(p_{2}, p_{3}\right)$ and $c_{3} \in\left(p_{3}, p_{1}\right)$, such that $f\left(c_{1}\right)=p_{1}, f\left(c_{2}\right)=p_{2}$ and $f\left(c_{3}\right)=p_{3}$.

(3) $f\left(\left[p_{1}, c_{1}\right]\right)=\left[p_{2}, p_{1}\right], f\left(\left[c_{1}, p_{2}\right]\right)=\left[p_{3}, p_{1}\right], f\left(\left[p_{2}, c_{2}\right]\right)=\left[p_{3}, p_{2}\right], f\left(\left[c_{2}, p_{3}\right]\right)=$ $\left[p_{1}, p_{2}\right], f\left(\left[p_{3}, c_{3}\right]\right)=\left[p_{1}, p_{3}\right], f\left(\left[c_{3}, p_{1}\right]\right)=\left[p_{2}, p_{3}\right]$.

Note that by construction, $1 \notin P(f)$. Let $n$ be any integer with $n>1$. Define $n$ closed intervals, $M_{1}, \ldots, M_{n}$, by $M_{1}=\left[p_{1}, c_{1}\right], M_{k}=\left[p_{2}, c_{2}\right]$ if $k$ is even and $2 \leqslant k \leqslant n$, and $M_{k}=\left[p_{3}, c_{3}\right]$ if $k$ is odd and $2 \leqslant k \leqslant n$. By Lemma 4, there is a fixed point $z$ of $f^{n}$ such that $z \in M_{1}, f(z) \in M_{2}, \ldots, f^{n-1}(z) \in M_{n}$. Since $\left[p_{1}, c_{1}\right]$ $\cap\left[p_{2}, c_{2}\right]=\varnothing$ and $\left[p_{1}, c_{1}\right] \cap\left[p_{3}, c_{3}\right]=\varnothing, z$ is a periodic point of $f$ of period $n$. Thus, $n \in P(f)$. Q.E.D.

Theorem C. Let $f \in C^{0}\left(S^{1}, S^{1}\right)$. If $\{1,2,3\} \subset P(f)$ then $P(f)=N$. Conversely, if $S \subset N$ with the property that for any $f \in C^{0}\left(S^{1}, S^{1}\right), S \subset P(f) \Rightarrow P(f)=N$, then $\{1,2,3\} \subset S$.

Proof. By Theorem A, if $\{1,2,3\} \subset P(f)$ then $P(f)=N$.

Suppose $S \subset N$ with the property that for any $f \in C^{0}\left(S^{1}, S^{1}\right), S \subset P(f) \Rightarrow$ $P(f)=N$. Since there are mappings $g$ of the interval into itself such that $3 \notin P(g)$ but $k \in P(g)$ for every positive integer $k \neq 3$ (see [3] or [4]), there are mappings $f \in C^{0}\left(S^{1}, S^{1}\right)$ such that $3 \notin P(f)$, but $k \in P(f)$ for every positive integer $k \neq 3$. Thus $3 \in S$. By Proposition 12 (with $n=3$ ), there is a map $f \in C^{0}\left(S^{1}, S^{1}\right)$ such that $1 \in P(f)$ and $3 \in P(f)$, but $2 \notin P(f)$. By Theorem A, for this map $f, P(f)$ consists of all positive integers except 2 . Thus, $2 \in S$. Finally, it follows from Lemma 13, that $1 \in S$. Hence $\{1,2,3\} \subset S$. Q.E.D. 


\section{REFERENCES}

1. L. Block, The periodic points of Morse-Smale endomorphisms of the circle, Trans. Amer. Math. Soc. 226 (1977), 77-88.

2. T. Li and J. A. Yorke, Period three implies chaos, Amer. Math. Monthly 82 (1975), 985-992.

3. A. N. Sarkovskii, Coexistence of cycles of a continuous map of a line into itself, Ukrain Mat. Ż. 16 (1964), 61-71.

4. P. Stefan, A theorem of Sarkouskii on the existence of periodic orbits of continuous endomorphisms of the real line, Comm. Math. Phys. 54 (1977), 237-248.

Department of Mathematics, University of Florida, Gainesville, Florida 32611 